\title{
Development of the Recursive Method of Continuation of Spectra in the Implementation of Super-Resolution Using Images of the Spacecraft Constellation Model
}

\author{
Viktor N. Vintaev ${ }^{1}$, Mikhail Y. Gilenev ${ }^{2}$, Natalya N. Ushakova ${ }^{1}$ \\ ${ }^{1}$ Belgorod University of Cooperation, Economics and Law, Belgorod, Russia \\ viktor.vn2010@yandex.ru,natush2006@yandex.ru \\ ${ }^{2}$ AO Corporation «VNIIEM», Moscow, Russia \\ zhilenev_mihail@bk.ru
}

\begin{abstract}
When implementing ultra-high resolution in the device grouping model, Kotelnikov's used theorem on image discretization and kernels of integral image deconvolution operators is aimed at spatial frequencies that lie far enough beyond the limits of transparency of the spatial frequency-contrast characteristic of the area-sensing virtual path. To further compensate for the loss of sharpness in the virtual channel due to dispersions in multiple overlay operations with images, the recursive method of extending the spatial-frequency spectra of images is implemented. The modified super-resolution method Iterative Back Projection for a group of images with a resolution of $1 \mathrm{~m}$ on the ground received patterns with a resolution of $0.5 \mathrm{~m}$ and further images were obtained with support for a resolution of $0.25 \mathrm{~m}$ (estimated by the Foucault method), which corresponds to the level of resolution for devices US intelligence. For a satellite with a resolution of $30 \mathrm{~cm}$, support of $15 \mathrm{~cm}$ is obtained.
\end{abstract}

Keywords: super resolution, deconvolution, subpixel shift, grouping of devices, function singular at measure zero, Kotelnikov theorem, continuation of the spectrum, Iterative Back Projection method 


\title{
РАЗРАБОТКА РЕКУРСИВНОГО МЕТОДА ПРОДОЛЖЕНИЯ СПЕКТРОВ ПРИ РЕАЛИЗАЦИИ СВЕРХРАЗРЕШЕНИЯ С ИСПОЛЬЗОВАНИЕМ ИЗОБРАЖЕНИЙ МОДЕЛИ ГРУППИРОВКИ КОСМИЧЕСКИХ АППАРАТОВ
}

\author{
В.Н. Винтаев ${ }^{1}$, М.Ю. Жиленев ${ }^{2}$, Н.Н. Ушакова ${ }^{1}$ \\ ${ }^{1}$ Белгородский университет кооперации, экономики и права, Белгород, Россия \\ viktor.vn2010@yandex.ru,natush2006@yandex.ru \\ ${ }^{2}$ АО Корпорация «ВНИИЭМ», Москва, Россия \\ zhilenev_mihail@bk.ru
}

\begin{abstract}
При реализации сверхвысокого разрешения в модели группировки аппаратов используемая теорема Котельникова о дискретизации изображений и ядер интегральных операторов деконволюции изображений нацеливается на пространственные частоты, лежащие достаточно далеко за границами прозрачности пространственной частотно-контрастной характеристики виртуального тракта зондирования ареала. Для дополнительной компенсации потерь резкости в виртуальном канале из-за дисперсий в многократных оверлейных операциях с изображениями реализуется рекурсивный метод продолжения пространственно-частотных спектров изображений. На модифицированном методе сверхразрешения Iterative Back Projection для группы изображений с разрешением в 1 м на местности получены паттерны с разрешением 0,5 м и далее получены изображения с поддержкой разрешения 0,25 м (оцененного по методу Фуко), что соответствует уровню разрешения для аппаратов видовой разведки США. Для спутника с реализацией разрешения в 30 см получена поддержка разрешения в 15 см.
\end{abstract}

Ключевые слова: сверхразрешение, деконволюция, субпиксельный сдвиг, группировка аппаратов, сингулярная на мере нуль функция, теорема Котельникова, продолжение спектра, метод Iterative Back Projection

\section{Введение}

Анализ формул оценки достижимого разрешения при дистанционном зондировании в оптическом диапазоне излучения позволяет использовать как пример приведенное ниже выражение [1,2]:

$$
L=M / 2 R=H K(Y, \beta) / 2 R f,
$$

где $L$ - измеряемое по методу Рэлея в единицах длины максимально возможное линейное разрешение на местности или апертура формируемого пиксела на ареале (с постоянным на нем уровнем яркости ); $M, H$ - соответственно, масштаб и высота наблюдения; $K(Y, \beta)$ - масштабный коэффициент при перспективном наблюдении; $Y$ - угол отклонения оптической оси от вертикали; $\beta$ - текущий угол поля изображения; $R$ - разрешающая способность носителя информации (вычисляемая по методу Фуко по верхней пространственной частоте изображения на носителе или определяемая, например, с помощью мирр); $f$ - фокусное расстояние аппаратуры наблюдения космического аппарата (КА).

Формула (1), если достигнуто соответствие значения $R$ и разрешения, определяемого апертурами пикселов приемного транспаранта позволяет рассчитать размеры пиксела на Земле при заданных размерах пикселов на приборах с зарядовой связью (ПЗС) оптоэлектронного 
устройства или ставить задачу о достижении необходимого размера пикселов на ПЗС или на виртуальных паттернах с изображением при требуемых размерах пикселов на наблюдаемой поверхности. При этом в технологии сверхразрешения реализуется уменьшение значения $L$ за счет увеличения $R$. Однако следует еще учесть связанную с качеством изготовления элементов оптики и методами учета и компенсации ухудшающих качество изображения факторов при формировании изображения [3] частотно-контрастную характеристику (ЧКХ) аппаратуры формирования световых потоков (ЧКХ телескопа, например) КА. Ели полоса полезной «прозрачности» ЧКХ невелика, то улучшение разрешения за счет параметра $R$ может приводить только к уменьшению апертур пикселов на ареале с постоянством самого сюжета изображения и его детальности. Увеличение детальности возможно, по крайней мере, с начальной моды спада полезной «прозрачности» упоминаемой ЧКХ, если эта и последующие ее моды входят во множество мод, предшествующих вычисляемой по методу Фуко верхней пространственной частоты определяемой увеличением $R$.

Использование деконволюции на основе модификаций фильтров Винера-Тихонова [2] для коррекции резкости не дает возможности предсказать увеличение радиуса пространственночастотных спектров (ПЧС) корректируемых изображений к какой-либо выбранной для контроля итерации оператора деконволюции и приходится ограничиваться тем, что есть рабочий критерий прерывания итераций, при срабатывании которого можно провести измерение радиуса получаемого ПЧС. Для прогноза и выхода при коррекции резкости на задаваемый уровень для корректируемых изображений, особенно в режиме сверхразрешения требуется построение коррекции ПЧС изображения с достоверно прогнозируемой величиной продолжения спектра. Соответствующим средством может быть рекурсивная процедура на основе аддитивной коррекции резкости, с предварительным выбором стартового порядка обобщенного градиентного оператора.

\section{1. Модель группировки аппаратов и технология сверхразрешения}

На изображениях со спутника «Ресурс ДК» для трех его каналов формирования изображений высокого разрешения при орбитальном движении аппарата можно выявлять соответствующие субпиксельные сдвиги изображений в каналах и на базе модифицированного для группировок спутников метода сверхразрешения Iterative Back Projection (IBP)[4,5] получать паттерны с вдвое и даже вчетверо уменьшенными по апертуре пикселами.

Измеряемая на изображениях функция рассеяния точки (ФРТ) не всегда обладает полнотой спектрального представления $F(\Phi P T)$ ( $F$ - двумерное Фурье-преобразование) для всех восстанавливаемых по резкости объектов. Но минимизируя невязку $F(\Phi P T)$ с оптимизированной ЧКХ тракта зондирования (канала) введением частотно-зависимой добавки $v$ к $F(\Phi P T)$, удается получить широко используемый в коррекции резкости модифицированный фильтр ВинераТихонова и, соответственно, итеративный оператор деконволюции изображений [2].

ЧКХ синтезируется отношением ПЧС зарегистрированного (или предыдущего в итерациях) изображения к ПЧС корректируемого изображения, а оптимизация ЧКХ заключается в соответствующей предобработке этих изображений так, чтобы огибающую ЧКХ можно было приблизить к квазипрямоугольной форме окна пропускания пространственных мод ПЧС на любом азимутальном еe срезе, не вызывая перерождения деконволюции в процессы контрастирования изображения, визуально принимаемые за улучшение резкости.

Оптимизация ЧКХ строится на принципах Нэша и Парето на функционалах, сформулированных на требованиях: минимизации тенденции спада и роста аппликат ЧКХ вплоть до приближения к высшим спектральным модам; максимальной гладкости ЧКХ; наиболее крутого спада ЧКХ в области высшей спектральной моды тракта; вписывания огибающей спектрального представления наблюдаемого на изображении опорного ориентира в огибающую ЧКХ; не превышения в коррекции резкости значением порядка обобщенного градиентного оператора порога, при котором резидентно присутствуют глобальное 
контрастирование или выделение контуров в первом шаге коррекции - аддитивной коррекции резкости.

В ЧКХ из-за того, что используемый для синтеза ЧКХ ПЧС корректируемого изображения в знаменателе отношения спектров может иметь достаточное количество нулей могут возникать сингулярные значения, методы регуляризации которых приводят к близкому к «истине» решению, которое не может удовлетворить требованиям, предъявляемым к технологии сверхвысокого разрешения.

Используется метод представления упомянутых сингулярностей ЧКХ моделями обобщенных сингулярных на мере нуль функций с финитными спектрами, парциальная свертка изображений с которыми при их подстановке в конечную формулу итеративной коррекции резкости (формулу Ван Циттера) дает решение по деконволюции с минимально возможными возмущениями измерительных характеристик обрабатываемых изображений. При этом необходимо увеличивать частоту дискретизации изображений и ядер интегральных операторов в соответствии с теоремой Котельникова, выбирая ее из условия согласования с верхней модой ПЧС модели сингулярной на мере нуль функции и отслеживая взаимосвязь критериев сходимости итеративного оператора деконволюции изображения [2] с выявлением достигаемого значения резкости по Фуко.

Модели интервалов меры нуль представляют собой апертуры исходных пикселов, деленные на $K$, и $K \geq 7$. Начальное значение критерия $K$ уже обеспечивает необходимую точность совмещений и замеров сдвигов изображений, что нормативно соответствует зонам ПЧС не поддерживаемым никакими трактами зондирования, т.е. лежащим в области полного обнуления регулярных ненулевых сигналов. По выбранному значению $K$ определяется погрешность сходимости итеративного оператора деконволюции.

На рисунке 1а в масштабированном окне одного из исходных каналов аппарата «РесурсДК» представлен пиксел (с неотфильтрованной ФРТ), далее на рисунке 16 показан виртуальный канал со сверхразрешением с в два раза меньшими по апертуре пикселами на изображении, полученном на методе IBP. Виден яркий элемент на выделенном и ранее засвеченном пикселе (левый верхний субпиксел) - максимально засвечен вдвое меньший по апертуре пиксел. На рисунке 1в представлен состоящий из четырех субпикселов, увеличенный до размера исходного пиксел, являющийся субпикселом на рисунке 1б, и поддерживающий следующий этап сверхразрешения, выполненного на двух субпиксельно сдвинутых изображениях от предыдущего этапа сверхразрешения, соответствующий по формуле (1) поддержке 0,25 м разрешения на ареале. Визуально различить упомянутые четыре субпиксела сложно, т.к. зона «прозрачности» ЧКХ телескопа аппарата уже находится на спаде и не дает возможности это сделать.

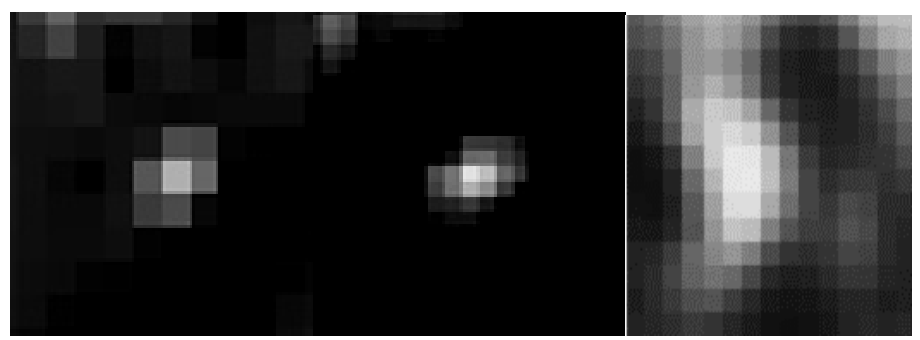

a)

б)

в)

Рис. 1. Пиксель: а) исходный и б) и в) субпиксельной мозаики изображения на первом и втором этапах сверхразрешения, соответственно

На рисунке 2 приведены исходное изображение с совмещением трех каналов без присвоения им кодов цветов палитры с ПЧС и со сверхразрешением с учетом субпиксельных сдвигов родительских изображений с ПЧС. 


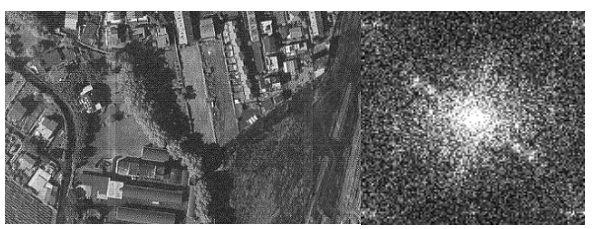

a)

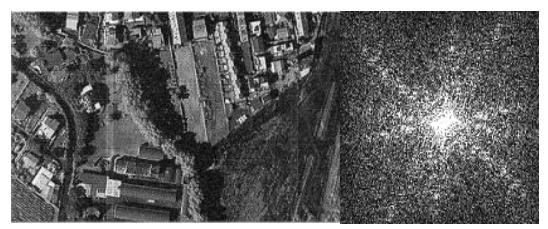

б)

Рис.2. а) - синтезированное по трем каналам панхроматическое изображение и его ПЧ и б) - окно после формирования сверхразрешения с вдвое меньшими по апертуре пикселами и ПЧС изображения

Радиус ПЧС изображения со сверхразрешением относительно радиуса ПЧС исходного панхроматического изображения увеличен в 1,7 раза. Это меньше, чем полученные на соответствующих моделях ранее результаты от 2 до 4 раз увеличения радиуса ПЧС и, соответственно, пространственного разрешения по Фуко [5]. На снижении профита технологии сверхразрешения сказывается отсутствие необходимого для двумерного паттерна четвертого независимого канала зондирования ареала и сам факт совмещения трех разнесенных пространственно, и, соответственно, разнесенных по требуемым параметрам изображений и неуправляемая дистанция между строками сформированных для дообработки изображений, что приводит к эллипсовидному годографу радиус-вектора значений разрешения с максимальной полуосью, направленной поперек трассовой составляющей орбиты спутника.

Используя для сверхразрешения каналы 1 и 2, и параллельно 2 и 3 получаем с субпиксельным сдвигом два изображения с потенциалом разрешения по строке в 50 см и на этих изображениях получаем далее вторым каскадом сверхразрешение по строке без присвоения цветовых кодов родительским изображениям, т.е. в панхроматическом режиме с потенциалом в 25 см, что соответствует диапазону разрешения, используемого в задачах видовой разведки аппаратами США. Результат второго каскада приведен на рисунке 3.

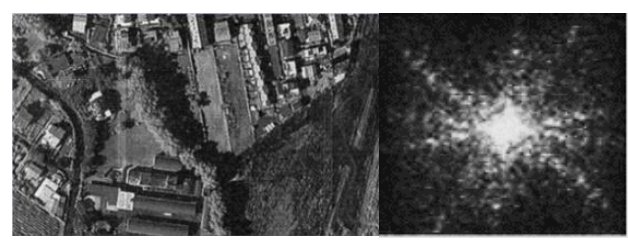

Рис. 3. Синтезированное по трем каналам панхроматическое изображение со сверхразрешением второго каскада и его ПЧС

В зависимости от оценки достоверности ФРТ на исходных изображениях итерации по деконволюции запускаются несколько раз с пошаговым изменением ФРТ из интервала доверительной оценки ФРТ и резкость в этом случае измеряется верхней и нижней мерами Лебега с присвоением выполненному итеративному оператору деконволюции значения дефекта между этими мерами в качестве оценки погрешности сходимости оператора. Существенной помехой при измерении субпиксельного сдвига пар изображений является смаз изображения (например, кинематический смаз первичных изображений большей или меньшей степени). Тем не менее, наилучшее качество изображения Земли, достижимое при применении данного конкретного аппарата, определяется статическими параметрами его съёмочной аппаратуры, факторами орбитальной съёмки и её геометрическими и радиометрическими условиями, что дает возможность прогноза на выполнение замеров субпиксельных сдвигов при совмещениях изображений для использования в дальнейшем их в технологии сверхразрешения [3].

\section{2. Аддитивная коррекция резкости и рекурсивный метод продолжения спектра изображения}

На базе прямого продолжения на вещественные значения порядка спектрального представления операции дифференцирования - $D_{x}^{\alpha}, D_{y}^{\beta}(0 \leq \alpha, \beta \leq 1)$, определяемого для 
допускающих Фурье-представление функций конструируем обобщенный градиентный оператор (фильтр) в виде нормы вектора $\left(D_{x}^{\alpha} S(x, y), D_{y}^{\beta} S(x, y)\right)$ т.е. в виде

$$
\operatorname{grad}_{\alpha \beta}(S)=\left(\left(D_{x}^{\alpha} S\right)^{2}+\left(D_{y}^{\beta} S\right)^{2}\right)^{1 / 2},
$$

где $S(x, y)$ - значение аппликаты изображения, заданного в плоскости $(x, y)$.

В соответствии с упоминаемой выше возможностью коррекции резкости, используя $\operatorname{grad}_{\alpha \beta}(S)$ в виде $\operatorname{grad}_{\alpha \alpha}(S)=\operatorname{grad}_{\alpha}(S)$ целесообразно реализовать коррекцию $S_{R}$ в виде аддитивной коррекции резкости [2]

$$
S_{U}=S_{R}+\operatorname{agrad}_{\alpha}\left(S_{R}\right)
$$

с варьируемыми параметрами $a$ и $\alpha$, т.е. коррекцию рекурсивным методом продолжения спектра, где $S_{R}-$ формируемое изображение; $S_{И}-$ восстанавливаемое изображение. Применяемая рекурсивно формула (3) к изображению характеризуется вычислимым однозначно продолжением спектра изображения на каждой итерации. Очевидно, что эффективный порядок оператора продолжения спектра (порядок «ближайшего» по норме дифференциального оператора с нецелым значением порядка) увеличивается с ростом числа рекурсий и тем быстрее, чем большим выбран стартовый порядок обобщенного градиентного оператора. Контроль за наклоном потолка огибающей ЧКХ формируемого таким образом тракта зондирования на итерациях для корректируемого изображения с предъявлением требования параллельности основанию или даже слабой деградации к высшим модам служит и средством останова, и средством формирования уверенности, что рекурсии не попали в зону перерождения процесса коррекции резкости в контрастирование.

В качестве тестовых примеров исследованы изображения со спутника «Ресурс-ДК» (Испания, г. Рота - фрагмент, 1м разрешения) и изображения со спутника OrbView-3 (30 см разрешения, фрагмент Houston_24466_0_8bit), используемого в видовой разведке США, но находящегося в «свободном доступе») [5].

Для спутника Ресурс ДК, например, оператор взаимного проектирования изображений в IBP определяет вычисленный двумерный субпиксельный сдвиг изображений с аппарата во всех трех каналах. Нормализация изображений реализуется выравниванием яркостных и геометрических невязок. На рисунке 4 для спутника OrbView-3 с поддержкой разрешения в 30 см представлена реализованная поддержка разрешения в 15 см.

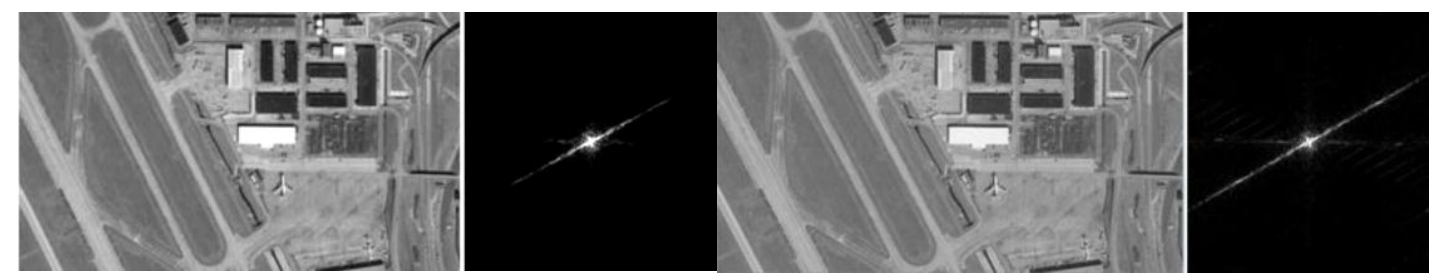

a)

б)

Рис.4. а) исходное изображение и его ПЧС, б) изображение со сверхразрешением и его ПЧС с инспирированием группировки аппаратов

\section{Заключение}

Метод рекурсивного продолжения спектра, применяемый для подавления остаточной ФРТ или ее существенного ослабления в технологии сверхразрешения проще метода синтеза итеративного оператора деконволюции. Построенный на принципе аддитивной коррекции резкости с вычислением этапов ЧКХ всего лишь для контроля наличия перерождения коррекции резкости в контрастирование изображения, метод отстает от результативности модернизированной Винеровской фильтрации с итеративным оператором деконволюции. 
Изображения дискретизируются и передискретизируются в соответствии с теоремой Котельникова, являются элементами конечномерного линейного, метрического Гильбертового пространства £ с евклидовой нормой; спектры интегральных операторов - интегралов свертки задаются проекциями их ядер на ортонормированный базис тригонометрических функций. В пространстве, сопряженном с £ дискретным преобразованием Фурье определяется градиентная операция с варьируемым нецелым порядком.

Исследование выполнено при финансовой поддержке РФФИ в рамках научного проекта №18-07-00201 и научного проекта № 19-07-00697.

\section{References}

[1] Malamed E.R. Designing space-based optical devices. St. Petersburg: GITMO, 2002, 368 p. (In Russian).

[2] Vintaev V.N., Ushakova N.N. Nontrivial correction of high resolution space images. Saarbrucken, Germany: Lambert Academic Publishing, 2018, 208 p. (In Russian).

[3] Makridenko L.A., Volkov S.N., Gecha V. Ya., Gilenev M. Y., Kazancev S.G. The main sources of the decrease in the quality of images of the earth obtained during orbital optical imaging from the ICA board// Electromechanical issues. Proceedings of VNIIEM. 2017. Vol. 160. -P. 3-19. (In Russian).

[4] Park S. C., Park M. K., Kang M. G. Super-resolution image reconstruction: A technical overview// IEEE Signal Processing Magazine. - 2003. - Vol. 20. - № 3. - P.21-36.

[5] Ushakova N.N. Mathematical model of the formation of cosmic image of high and very high resolution in the group of spacecraft// Belgorod State University Scientific Bulletin Mathematics \& Physics. 2016. № 20 (44). P. 155-167. (In Russian). 